\title{
THE MACKEY-GLEASON PROBLEM
}

\author{
L. J. BUNCE AND J. D. MAITLAND WRIGHT
}

\begin{abstract}
Let $A$ be a von Neumann algebra with no direct summand of Type $\mathrm{I}_{2}$, and let $\mathscr{P}(A)$ be its lattice of projections. Let $X$ be a Banach space. Let $m: \mathscr{P}(A) \rightarrow X$ be a bounded function such that $m(p+q)=m(p)+m(q)$ whenever $p$ and $q$ are orthogonal projections. The main theorem states that $m$ has a unique extension to a bounded linear operator from $A$ to $X$. In particular, each bounded complex-valued finitely additive quantum measure on $\mathscr{P}(A)$ has a unique extension to a bounded linear functional on $A$.
\end{abstract}

\section{Physical BaCKGROUND}

In von Neumann's approach to the mathematical foundations of quantum mechanics, the bounded observables of a physical system are identified with a real linear space, $L$, of bounded selfadjoint operators on a Hilbert space $H$. It is reasonable to assume that $L$ is closed in the weak operator topology and that whenever $x \in L$ then $x^{2} \in L$. (Thus $L$ is a Jordan algebra and contains spectral projections.) Then the projections in $L$ form a complete orthomodular lattice, $\mathscr{P}$, otherwise known as the lattice of "questions" or the quantum logic of the physical system. A quantum measure is a map $\mu: \mathscr{P} \rightarrow \mathbb{R}$ such that whenever $p$ and $q$ are orthogonal projections $\mu(p+q)=\mu(p)+\mu(q)$.

In Mackey's formulation of quantum mechanics [11] his Axiom VII makes the assumption that $L=L(H)_{s a}$. Mackey states, that in contrast to his other axioms, Axiom VII has no physical justification; it is adopted for mathematical convenience. One of the technical advantages of this axiom was that, by Gleason's Theorem, a completely additive positive quantum measure on the projections of $L(H)$ is the restriction of a bounded linear functional (provided $H$ is not two-dimensional). In order to weaken Axiom VII it was desirable to strengthen Gleason's Theorem.

\section{INTRODUCTION}

Let $\mathscr{P}(A)$ be the lattice of projections in a von Neumann algebra $A$, let $X$ be a Banach space, and let $\mu: \mathscr{P}(A) \rightarrow X$ be a function such that

(a) $\mu(e+f)=\mu(e)+\mu(f)$ whenever $e f=0$,

(b) $\sup \{\|\mu(e)\|: e \in \mathscr{P}(A)\}<\infty$.

Then $\mu$ is said to be a finitely additive, $X$-valued measure on $\mathscr{P}(A)$.

Clearly each bounded linear operator from $A$ to $X$ restricts to a finitely additive $X$-valued measure. When $A$ is the algebra of two-by-two matrices and $X$ is one-dimensional, there exist examples of measures that fail to extend to linear functionals.

Received by the editors May 21, 1991.

1991 Mathematics Subject Classification. Primary 46L50. 
Our main result is

Theorem A. Let $A$ be a von Neumann algebra with no direct summand of Type $I_{2}$. Then, for each Banach space $X$, each $X$-valued measure on $\mathscr{P}(A)$ has $a$ unique extension to a bounded linear operator from $A$ to $X$.

This immediately specializes to give

Theorem B. Let $A$ be a von Neumann algebra with no direct summand of Type $\mathrm{I}_{2}$. Then each complex-valued finitely additive measure on $\mathscr{P}(A)$ extends to a bounded linear functional on $A$.

In fact, we shall see that Theorem A follows easily from Theorem B. We note, however, that to deduce Theorem A it is essential to have Theorem B for all real-valued finitely additive measures. It does not suffice to know this result for positive measures or for countably additive measures. The lack of positivity causes considerable difficulty in establishing this theorem.

Theorem B answers a natural question first posed by G. W. Mackey some thirty years ago. When $\mu$ is positive, that is, when $\mu(e) \geq 0$ for each projection $e$. Theorem B was established by Christensen [7] for properly infinite algebras and algebras of Type $I_{n}$ and by Yeadon $[15,16]$ for algebras of finite type. The first major progress had been made by Gleason [9], who, by using an ingenious geometric argument, settled the question for positive completely additive measures on the projections of $L(H)$. (See [8] for an elementary proof of this deep result.) Aarnes [1] and Gunson [10] made important contributions, especially concerning continuity properties. Paszkiewicz [13], working independently of Christensen and Yeadon, established Theorem B for $\sigma$-finite factors, however, to extend his results to nonfactorial von Neumann algebras, he requires $\mu$ to be positive and countably additive. A lucid and meticulous exposition of Theorem B for positive measures is given by Maeda [12].

\section{VECTOR MEASURES}

The following short argument shows that Theorem $\mathrm{A}$ is a consequence of Theorem B.

Lemma 1.1. Let $A$ be a von Neumann algebra such that each finitely additive (complex) measure on $\mathscr{P}(A)$ has an extension to a bounded linear functional. Let $X$ be a Banach space and let $m: \mathscr{P}(A) \rightarrow X$ be a finitely additively $X$ valued measure. Then $m$ has a unique extension to a bounded linear operator from $A$ to $X$.

Proof. Elementary estimates show that when $\beta \in A^{*}$ then

$$
\|\beta\| \leq 4 \sup \{|\beta(p)|: p \in \mathscr{P}(A)\} .
$$

Let $K$ be a constant such that $\|m(p)\| \leq K$ for each $p \in \mathscr{P}(A)$.

For any $\phi \in X^{*}, p \rightarrow \phi m(p)$ is a complex-valued finitely additive measure on $\mathscr{P}(A)$. By hypothesis there exists $\beta \in A^{*}$ such that $\beta(p)=\phi m(p)$ for each $p \in \mathscr{P}(A)$. Then

Let $x=\sum_{1}^{n} \lambda_{j} p_{j}$ be a finite linear combination of projections $p_{1}, p_{2}, \ldots, p_{n}$.

$$
\phi\left(\sum_{1}^{n} \lambda_{j} m\left(p_{j}\right)\right)=\sum_{1}^{n} \lambda_{j} \phi m\left(p_{j}\right)=\beta\left(\sum_{1}^{n} \lambda_{j} p_{j}\right)=\beta(x) .
$$


So

$$
\begin{aligned}
\left|\phi\left(\sum_{1}^{n} \lambda_{j} m\left(p_{j}\right)\right)\right| & \leq 4\|x\|\|\phi\| \sup \{\|m(p)\|: p \in P(A)\} \\
& \leq 4\|x\|\|\phi\| K .
\end{aligned}
$$

It follows from the Hahn-Banach Theorem that

$$
\left\|\sum_{1}^{n} \lambda_{j} m\left(p_{j}\right)\right\| \leq 4 K\|x\| \text {. }
$$

In particular, $\sum_{1}^{n} \lambda_{j} p_{j}=0$ implies $\sum_{1}^{n} \lambda_{j} m\left(p_{j}\right)=0$. So $m$ has a unique extension to a linear operator $T: \operatorname{Span} \mathscr{P}(A) \rightarrow X$.

$$
\left\|T\left(\sum_{1}^{n} \lambda_{j} p_{j}\right)\right\|=\left\|\sum_{1}^{n} \lambda_{j} m\left(p_{j}\right)\right\| \leq 4 K\left\|\sum_{1}^{n} \lambda_{j} p_{j}\right\| .
$$

So $T$ is bounded and hence has a unique extension to a bounded linear operator from $A$ to $X$.

\section{SCAlar measures}

In all that follows $A$ is a von Neumann algebra with no direct summand of Type $\mathrm{I}_{2}$. Let $\mathscr{P}(A)$ be the lattice of projections in $A$.

Since each complex-valued measure on $\mathscr{P}(A)$ is of the form $\mu+i \nu$, where $\mu$ and $\nu$ are real-valued measures, it suffices to prove Theorem B for real-valued measures.

From now onward, $\mu$ is a finitely additive real-valued measure on $\mathscr{P}(A)$. That is, $\mu: \mathscr{P}(A) \rightarrow \mathbb{R}$ is a function such that

(a) $\mu(p+q)=\mu(p)+\mu(q)$ whenever $p$ and $q$ are orthogonal projections;

(b) $\sup \{|\mu(p)|: p \in \mathscr{P}(A)\}<+\infty$.

We define the variation of $\mu, V$, by

$$
V(p)=\sup \{|\mu(e)|: e \leq p\}
$$

we also define

$$
\alpha(p)=\sup \{\mu(e): e \leq p\} .
$$

Straightforward arguments show that $\mu$ has a unique extension to a function $\bar{\mu}: A \rightarrow \mathbb{C}$, where $\bar{\mu}$ is linear and bounded on each abelian ${ }^{*}$-subalgebra of $A$ and where $\bar{\mu}(x+i y)=\bar{\mu}(x)+i \bar{\mu}(y)$ whenever $x$ and $y$ are selfadjoint. Moreover, it can be shown [4] that

(1) $\sup \left\{|\bar{\mu}(x)|: x=x^{*}\right.$ and $\left.\|x\| \leq 1\right\}=2 \alpha(1)-\mu(1)$,

(2) $\sup \{\bar{\mu}(x): 0 \leq x \leq 1\}=\alpha(1)$.

In order to see (2), let $0 \leq x \leq 1$. Then, for suitable spectral projections, $x=\sum_{1}^{\infty} 2^{-n} e_{n}$. Hence, for some $n, \mu\left(e_{n}\right) \geq \bar{\mu}(x)$.

The lack of positivity of $\mu$ greatly increases the difficulties of establishing the linearity of $\bar{\mu}$ when $A$ is properly infinite or when $A$ is of Type $\mathrm{II}_{1}$. However, when $A$ is of Type $\mathrm{I}_{n}(n \neq 2)$ linearity can be established by a fairly straightforward extension of the arguments for positive measures.

The first step is to notice that when $\mu$ is a measure on the projections of $M_{n}(\mathbb{C})$, the algebra of $n \times n$ matrices over $\mathbb{C}$, then when $T$ is the canonical 
(unnormalized) trace on $M_{n}(\mathbb{C})$ we have $\mu(e) \leq \alpha(1)=\alpha(1) T(e)$ for each minimal projection $e$. So $\alpha(1) T-\mu$ is a positive measure on $\mathscr{P}\left(M_{n}(\mathbb{C})\right)$. Hence, by Gleason's Theorem for finite-dimensional Hilbert spaces, $\alpha(1) T-$ $\bar{\mu}$ is linear (provided $n \neq 2$ ). We now revert to the general situation and conclude that $\bar{\mu}$ is linear on each subalgebra of $A$ that can be embedded in a subalgebra of $A$ that is isomorphic to a Type $\mathrm{I}_{n}$ factor $(n \geq 3)$. By elementary algebraic arguments, it can be shown [8] that if $B$ is a subalgebra of $A$ and $B \approx M_{2}(\mathbb{C}) \subset \mathbb{D}$, then either $B \subset C \subset A$, where $C \approx M_{4}(\mathbb{C})$ or $B \subset C \oplus D$, where $C \simeq M_{4}(\mathbb{C})$ and $D \subset E \subset A$ with $E \approx M_{3}(\mathbb{C})$. Hence $\bar{\mu}$ is linear on $B$.

By "patching" together Type $\mathrm{I}_{2}$ factorial subalgebras of $A$ it can be shown [2], building on techniques of Christensen [7], that $\mu$ is uniformly continuous on $\mathscr{P}(A)$ and $\bar{\mu}$ is linear on each Type $\mathrm{I}_{n}$ subalgebra of $A$. In particular, $\bar{\mu}$ is linear on $W(1, p, q)$, where $W(1, p, q)$ is the $W^{*}$-subalgebra of $A$ generated by the identity and an arbitrary pair of projections $p$ and $q$.

\section{APPROXIMATE LINEARITY}

The restriction of $\bar{\mu}$ to the centre of $A$ is linear. So, by the Hahn-Banach Theorem, there exists $\sigma \in A^{*}$ such that $\bar{\mu}$ and $\sigma$ coincide on the centre. By replacing $\bar{\mu}$ by $\bar{\mu}-\sigma$ if necessary, we can assume that $\bar{\mu}$ vanishes on the centre of $A$. By dividing by a suitable constant we can also assume that $\sup \left\{|\bar{\mu}(x)|: x=\lambda^{*},\|\lambda\| \leq 1\right\}=1$. It can then be shown that $\alpha(1)=\frac{1}{2}$.

Lack of positivity leads to a number of difficulties establishing

Lemma 3.1. Let $A$ be properly infinite. Let $0<\delta<\frac{1}{2}$. There exists a projection $e$ in $A$ with $1 \sim e \sim 1-e$ and such that $\frac{1}{2}-\delta^{2}<\mu(e)$. Then, for each $p \in \mathscr{P}(A)$.

$$
|\mu p-\bar{\mu}(e p e)-\bar{\mu}(1-e) p(1-e)|<5 \delta .
$$

The next lemma depends on the fact that $\bar{\mu}$ is linear on each $W^{*}$-subalgebra generated by a pair of projections.

Lemma 3.2. Let $0<\varepsilon<1$ and let $m$ be such that $\sum_{m+1}^{\infty} 2^{-n}<\varepsilon$. Let $e$ be $a$ projection such that

(1) $\mid \mu(p)-\bar{\mu}($ epe $)-\bar{\mu}((1-e) p(1-e)) \mid<\varepsilon / m$ for each $p \in \mathscr{P}(A)$;

(2) $|\bar{\mu}(a+b)-\bar{\mu}(a)-\bar{\mu}(b)|<\varepsilon / m$ whenever $a \geq 0, b \geq 0$, and $a+b \leq e$;

(3) $|\bar{\mu}(c+d)-\bar{\mu}(c)-\bar{\mu}(d)|<\varepsilon / m$ whenever $c \geq 0, d \geq 0$, and $c+d \leq 1-e$. Then, whenever $x \geq 0, y \geq 0$, and $x+y \leq 1,|\bar{\mu}(x+y)-\bar{\mu}(x)-\bar{\mu}(y)|<20 \varepsilon$.

We shall now sketch a proof of Theorem B for $A$ a properly infinite von Neumann algebra.

Let $a$ and $b$ be fixed, positive elements of $A$ with $a+b \leq 1$. Choose $\varepsilon$ with $0<\varepsilon<1$. Let $m$ be such that $\sum_{m+1}^{\infty} 2^{-n}<\varepsilon$. We put $\delta=\varepsilon / 42 m$. Let $e$ be a projection that satisfies the conditions of Lemma 3.1.

Let $x \geq 0, y \geq 0$ with $x+y \leq e$.

We find a projection $f \leq 1-e$ such that $f \sim 1$ and $V(f)<\delta^{2}$. Put $h=e+f$. We then find three orthogonal projections, majorized by $f$ and each equivalent to $e$. By applying the $4 \times 4$ matrix construction due to Christensen [7] we can find orthogonal projections $p$ and $q$, majorized by $h=e+f$, with $x=2 e p e$ and $y=2 e q e$. Since $x$ is in the ${ }^{*}$-algebra generated by $e$ and $p$,

$$
|2 \mu(p)-\bar{\mu}(x)|=|\bar{\mu}(2 p-x)|=2|\bar{\mu}(p-e p e)| \text {. }
$$


Since $e, f$, and $p$ are in the properly infinite $W^{*}$-algebra $h A h$ and $e+f=h$, we see that $e$ is in the $W^{*}$-subalgebra of $h A h$ generated by $f$ and $p$. So

$$
2|\bar{\mu}(p-e p e)| \leq 2|\bar{\mu}(f p f)|+2|\bar{\mu}(f p e+e p f)| \text {. }
$$

By considering $\bar{\mu}$ restricted to $f A f$ we can show that

$$
|\mu(f p f)| \leq 2 V(f)<2 \delta^{2} .
$$

Also,

$$
f p e+e p f=(1-e) p e+e p(1-e) .
$$

So, applying Lemma $3.1,|\bar{\mu}(f p e+e p f)|<5 \delta$. So

$$
|2 \mu(p)-\bar{\mu}(x)|<2 \delta^{2}+10 \delta<14 \delta .
$$

Similarly,

So

$$
|2 \mu(q)-\bar{\mu}(y)|<14 \delta \text { and }|2 \mu(p+q)-\bar{\mu}(x+y)|<14 \delta .
$$

$$
|\bar{\mu}(x+y)-\bar{\mu}(x)-\bar{\mu}(y)|<42 \delta=\frac{\varepsilon}{m} .
$$

We may repeat the above argument, interchanging the roles of $e$ and $1-e$, to deduce that whenever $z \geq 0, w \geq 0$, and $z+w<1-e$,

$$
|\bar{\mu}(w+z)-\bar{\mu}(w)-\bar{\mu}(z)|<\frac{\varepsilon}{m} .
$$

We now appeal to Lemma 3.2 to obtain

$$
|\bar{\mu}(a+b)-\bar{\mu}(a)-\bar{\mu}(b)|<20 \varepsilon .
$$

Since $\varepsilon$ is arbitrary, it follows that

$$
\bar{\mu}(a+b)=\bar{\mu}(a)+\bar{\mu}(b) .
$$

Hence $\bar{\mu}$ is linear.

When $\mu$ is $\sigma$-additive, we can give a reasonably straightforward proof of linearity for Type $\mathrm{II}_{1}$ algebras. However, in order to obtain Theorem $\mathrm{A}$, it is essential to obtain Theorem B when $\mu$ is finitely additive, not positive and not $\sigma$-additive. For von Neumann algebras of finite type this forces us to use a more elaborate argument.

\section{OPEN PROBLEM}

Let $A$ be a (unital) $C^{*}$-algebra. Let $\phi: A \rightarrow \mathbb{C}$ be a function whose restriction to each abelian ${ }^{*}$-subalgebra is linear, is such that $\{|\phi(x)|:\|x\| \leq 1\}$ is bounded and whenever $x$ and $y$ are selfadjoint, $\phi(x+i y)=\phi(x)+i \phi(y)$. Then $\phi$ is said to be a quasi-linear functional.

Problem. For which $C^{*}$-algebras $A$ is it true that every quasi-linear functional on $A$ is linear?

We have the following consequence of Theorem B.

Corollary. Let $M$ be a von Neumann algebra with no direct summand of Type $\mathrm{I}_{2}$. Let $I$ be a closed ideal of $M$ and let $A=M / I$. Then every quasi-linear functional on $A$ is linear.

Proof. Let $\phi: A \rightarrow \mathbb{C}$ be quasi-linear. Let $\pi: M \rightarrow M / I$ be the canonical quotient homomorphism. Then the restriction of $\phi \pi$ to $\mathscr{P}(M)$ is a finitely additive measure that, by Theorem $\mathrm{B}$, has a unique extension to a bounded linear functional on $M$. Hence $\phi$ is linear.

Corollary. Each quasi-linear functional on the Calkin algebra is linear. 


\section{REFERENCES}

1. J. F. Aarnes, Quasi-states on $C^{*}$ algebras, Trans. Amer. Math. Soc. 149 (1970), 601-625.

2. L. J. Bunce and J. D. M. Wright, Complex measures on projections in von Neumann algebras, J. London Math. Soc. (2) (to appear).

3. Continuity and linear extensions of quantum measures on Jordan operator algebras, Math. Scand. 64 (1989), 300-306).

4. _ The Mackey-Gleason problem for vector measures on projections in a von Neumann algebra, submitted.

5. __ Quantum logic, state space geometry and operator algebras, Comm. Math. Phys. 96 (1984), 345-348.

6. __ Quantum measures and states on Jordan algebras, Comm. Maths. Phys. 98 (1985), 187-202.

7. E. Christensen, Measures on projections and physical states, Comm. Math. Phys. 86 (1982), 529-538.

8. R. Cooke, M. Keane, and W. Moran, An elementary proof of Gleason's Theorem, Math. Proc. Cambridge Philos. Soc. 98 (1985), 117-128.

9. A. M. Gleason, Measures on the closed subspaces of a Hilbert space, J. Math. Mech. 6 (1957), 885-893.

10. J. Gunson, Physical states on quantum logics I, Ann. Inst. H. Poincaré 17 (1972), 295-311.

11. G. W. Mackey, The mathematical foundations of quantum mechanics, Benjamin, 1963.

12. S. Maeda, Probability measures on projections in von Neumann algebras, Reviews in Mathematical Physics 1 (1990), 235-290.

13. A. Paszkiewicz, Measures on projections in $W^{*}$-factors, J. Funct. Anal. 62 (1985), 87-117.

14. M. Takesaki, Theory of operator algebras, Springer, 1979.

15. F. J. Yeadon, Finitely additive measures on projections in finite $W^{*}$-algebras, Bull. London Math. Soc. 16 (1984), 145-150.

16. _ Measures on projections in $W^{*}$-algebras of Type $\mathrm{II}_{1}$, Bull. London Math. Soc. 15 (1983), 139-145.

Analysis and Combinatorics Research Centre, Mathematics Department, University of Reading, P O Box 220, Whiteknights, Reading RG6 2AX, England 